\title{
Public opinion on global rollout of COVID-19 vaccines
}

To the Editor - Vaccination programs for COVID-19 in 'high-income countries' (HICs) have benefited from their ability to secure contracts for preferential supply for several vaccines ${ }^{1}$. For the rest of the world, vaccine access is much less certain. Although leaders of G20 nations have pledged to ensure fair distribution of vaccines against COVID-19 worldwide, substantial challenges remain. The COVAX Facility aims to ensure that all countries will have equal access to doses and can compete with HICs to acquire doses, but some low-income countries may need to wait until at least 2022 before even the most vulnerable $20 \%$ of their populations are vaccinated ${ }^{2}$.

There are increasing calls for HICs to donate a proportion of their vaccine doses. During the H1N1 influenza pandemic, there was a coordinated effort among HICs to make a vaccine to protect the world's poorest, including a pledge by US President Obama to donate $10 \%$ of the USA's vaccine supply; this was supported by the general public ${ }^{3}$.

A key factor that could shape the willingness of governments to make vaccines against COVID-19 available to low-income countries is their public's willingness to support donations. We provide evidence on this issue obtained through an international internet-based survey ${ }^{4}$ that was conducted between 24 November and 28 December 2020. The overall study involved 15,536 people from 13 countries who completed an anonymous survey using Qualtrics web-based software. Quota sampling (and, in five countries, additional weighting) was (were) used to obtain a sample that reflected the distribution of age, education, gender and region in each country.
We obtained information from 8,209 adults from a subset of seven HICs (Australia, Canada, France, Italy, Spain, UK and USA). The survey used a visual analog scale to measure agreement (from 0 ('very much disagree') to 100 ('very much agree')), with three prioritization principles for the global allocation of treatments for and vaccines against COVID-19. We asked if these should be first provided for (i) "those who need them most"; (ii) "those who cannot afford to buy them"; and (iii) "those who live in the country in which they are first developed". Second, we adapted a question previously used in the context of H1N1 influenza ${ }^{3}$ and asked whether respondents supported donating some doses of vaccines against COVID19 for distribution to poor countries with insufficient resources to buy their own vaccines. Those willing to donate indicated whether they favored an amount greater than, equal to or less than $10 \%$ of their country's doses. Proportions and means, including 95\% confidence intervals (CIs), are reported, and the data used to estimate these statistics are available from the authors upon request.

For the global allocation criteria for vaccines, the highest average level of agreement was based on need (with average agreement ranging from $70(95 \% \mathrm{CI}$, $68-71)$ to 80 (95\% CI, 78-81)); followed by affordability (62 (95\% CI, 60-64) to 70 (95\% CI, 68-71)); and finally whether the country developed the vaccine $(28$ (95\% CI, $26-29)$ to 58 (95\% CI, 56-60)). This ranking was consistent across all countries. We present here the opinions on the donation of purchased vaccines (Table 1). The proportion of people supporting donation was more than double the proportion of those who did not support such donation.

While current supplies are limited, many HICs have pre-purchased supplies that exceed their population size. For example, the USA has reserved more than 1.2 billion doses ${ }^{5}$, and Canada has pre-market commitments covering more than nine doses per person ${ }^{1}$. Redistributing some of these supplies would have global benefits. It would reduce the risk of the emergence and spread of new variants and, according to predictions, would benefit the economy, both globally and in donor countries ${ }^{6}$. In contrast, the economic cost of 'vaccine nationalism' (whereby a few countries push to gain preferential access) is potentially high, with a recent report suggesting it could cost up to US $\$ 1.2$ trillion per year of the world's economy ${ }^{7}$

Although we did not investigate opinions on the timing of donations, national vaccination strategies should be taking into account these broader benefits. Notably, countries such as Norway ${ }^{8}$ have already pledged to distribute vaccines at the same time that they vaccinate their own populations. Such policies are likely to maximize global health benefits. A recent modeling study suggests that allocating doses internationally in proportion to countries' population sizes would be a close-to-optimal strategy in terms of averting deaths?.

Like prevalence studies, these opinions represent a single point in time, but, notably, our survey was conducted at a time when the distribution of vaccines against COVID19 was no longer a hypothetical question.

Table 1 | Preferences of the public for the donation of vaccines governments have purchased, by country

\begin{tabular}{|c|c|c|c|c|c|c|c|}
\hline \multirow[t]{2}{*}{ Country } & \multicolumn{4}{|c|}{ Willing to donate } & \multirow{2}{*}{$\begin{array}{l}\text { Not willing to } \\
\text { donate }\end{array}$} & \multirow[t]{2}{*}{ Do not know } & \multirow[t]{2}{*}{ Prefer not to say } \\
\hline & $<10 \%$ & $10 \%$ & $>10 \%$ & Any level & & & \\
\hline Australia & $10(8-12)$ & $21(18-23)$ & $20(18-22)$ & $51(48-54)$ & $20(17-22)$ & $27(24-29)$ & $3(2-4)$ \\
\hline Canada & $15(13-17)$ & $26(23-28)$ & $15(13-18)$ & $56(53-59)$ & $20(18-23)$ & $22(19-24)$ & $2(1-3)$ \\
\hline France & $11(9-13)$ & $16(14-19)$ & $21(18-23)$ & $48(45-51)$ & $20(17-22)$ & $28(25-31)$ & $4(3-5)$ \\
\hline Italy & $13(11-15)$ & $19(16-21)$ & $22(19-25)$ & $54(50-57)$ & $15(13-18)$ & $28(25-31)$ & $3(2-4)$ \\
\hline Spain & $11(9-13)$ & $25(23-28)$ & $18(16-21)$ & $55(52-58)$ & $15(13-17)$ & $23(21-26)$ & $7(5-8)$ \\
\hline UK & $14(12-16)$ & $21(19-24)$ & $16(13-18)$ & $51(48-54)$ & $26(23-29)$ & $22(19-24)$ & $1(1-2)$ \\
\hline USA & $10(8-12)$ & $19(16-22)$ & $22(20-25)$ & $52(48-55)$ & $17(15-20)$ & 25 (22-28) & $6(4-8)$ \\
\hline
\end{tabular}

Amounts for 'Willing to donate' ( $<10 \%, 10 \%,>10 \%)$ are the percentage of the country's doses; the column 'Any level' combines responses from those three categories. Results include $95 \%$ Cl in parentheses. Sample sizes: Australia, 1,364; Canada, 1,150; France, 1,145; Italy, 1,081; Spain, 1,153; UK, 1,165; USA, 1,150. Based on data from the CANDOUR study (details, https://oxford-candour.com/). 
More broadly, understanding and potentially influencing public opinion will be important components of any strategies to combat COVID-19 and prevent future pandemics ${ }^{10}$. This global problem requires global solutions, and our survey indicates that the redistribution of some pre-purchased vaccines to countries most in need has public support.

\section{Data Availability}

Data are available from the authors upon request.

\section{Editorial note: This article has been peer-reviewed.}

\section{Philip M. Clarke (D) ${ }^{凶}$, Laurence S. J. Roope (iD) Peter John Loewen ${ }^{2}$, Jean-Francois Bonnefon ${ }^{3}$, Alessia Melegaro (D) 4 , Jorge Friedman ${ }^{5}$, Mara Violato ${ }^{1}$, Adrian Barnett ${ }^{6}$ and Raymond Duch (D) \\ ${ }^{1}$ Health Economics Research Centre, Nuffield Department of Population Health, University of Oxford, Oxford, UK. ${ }^{2}$ Department of Political Science and the Munk School of Global Affairs \& Public Policy, University of Toronto, Toronto, Ontario, Canada. ${ }^{3}$ Toulouse School of Economics, Toulouse, France. ${ }^{4}$ Dondena Centre for Research on Social}

Dynamics and Public Policy, Department of Social and Political Sciences, Bocconi University, Milan, Italy. ${ }^{5}$ Facultad de Administración y Economía, Universidad de Santiago de Chile, Santiago, Chile. ${ }^{6}$ Queensland University of Technology, Brisbane, Queensland, Australia. ${ }^{7}$ Nuffield College, University of Oxford, Oxford, UK.

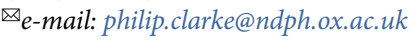

Published online: 30 March 2021

https://doi.org/10.1038/s41591-021-01322-9

References

1. Mullard, A. Nature https://www.nature.com/articles/d41586-02003370-6 (2020).

2. Nature 589, 170 (2021).

3. Kumar, S., Quinn, S. C., Kim, K. H. \& Hilyard, K. M. PLoS One 7, e33025 (2012).

4. Duch, R. et al. Preprint at https://www.medrxiv.org/content/10.11 01/2021.01.31.21250866v1 (2021).

5. Duke Global Health Innovation Center. https:// launchandscalefaster.org/COVID-19 (accessed 9 March 2020).

6. Çakmakl, C., Demiralp, S., Kalemli-Özcan, S., Yeşiltaş, S. \& Ylldırım, M.A. National Bureau of Economic Research https://doi. org/10.3386/w28395 (2021).

7. Hafner, M., Yerushalmi, E., Fays, C., Dufresne, E. \& Van Stolk, C. RAND Corporation https://www.rand.org/pubs/research_reports/ RRA769-1.html (2020).

8. Rigby, J. \& Newey, S. The Telegraph https://www.telegraph. co.uk/global-health/science-and-disease/norway-share-covid19-vaccine-poorer-countries-time-protecting/ (20 January 2021).

9. Hogan, A.B. et al. Imperial College London https://doi. org/10.25561/82822 (25 September 2020).

10. Bonell, C. et al. J. Epidemiol. Community Health 74, 617-619 (2020).

\section{Acknowledgements}

The research was supported by funding from the National Institute for Health Research (NIHR) Oxford Biomedical Research Centre and the Department of Health and Social Care for evaluation of the COVID-19 Oxford Vaccine Trial. The views expressed are those of the author(s) and not necessarily those of the NHS, the NIHR or the Department of Health and Social Care. We acknowledge the support of the Office of the Dean of the Faculty of Arts \& Science at the University of Toronto. J.-F.B. acknowledges support from the grant ANR-17-EURE-0010 Investissements d'Avenir. J.F. received funding from the University of Santiago project Dicyt USA1899. A.M. acknowledges support from the Italian Ministry of Education Progetti di Rilevante Interesse Nazionale, grant number 20177BRJXS M.V. receives funding from the NIHR Applied Research Collaboration Oxford and Thames Valley at Oxford Health NHS Foundation Trust. R.D. acknowledges support from the grant FONDECYT No1201397. The survey study was approved by the University of Oxford Medical Sciences Interdivisional Research Ethics Committee (approval ID: R72328/RE001).

Author contributions

P.M.C. and R. D., overall design of the CANDOUR study; L.S.J.R., P.J.L. and M.V., design of the questionnaire; J.-F.B., A.M. and J.F., interpretation of the results; and A.B., statistical analysis. All authors contributed to the manuscript and approved the final version.

\section{Improving patient care through the development of a 5G-powered smart hospital}

To the Editor-In 2018, Guangdong Second Provincial General Hospital (GD2H) started incorporating artificial intelligence (AI) into hospital management and operations, including patient registration and triage, diagnosis aids, health-record organization, digital payment, and the transportation of operating-room supplies $^{1,2}$. Due to the limitations of big-data sharing via the current $4 \mathrm{G}$ hospital network, practical applications of AI cannot be closely connected throughout the hospital, which compromises efficiency and reduces patient satisfaction. For example, if a patient is waiting for emergency surgery in the operating room after a magnetic resonance imaging scan, the surgeons can proceed only until the image files and reports are transferred to the system in the operating room, which takes time and causes delays.
To address this issue, GD2H recently announced the building of a comprehensive smart hospital in conjunction with Huawei, using $5 \mathrm{G}$ technology that features low latency, high capacity, increased bandwidth and a wireless nature ${ }^{3}$. The $5 \mathrm{G}$ hospital has attracted worldwide attention because of the potential for fundamentally changing how hospitals operate. By using $5 \mathrm{G}$ in combination with cloud storage and AI, the comprehensive $5 \mathrm{G}$ smart hospital will cover areas of healthcare, teaching and training, research, and management, with $5 \mathrm{G}$ technology applied both within and outside the hospital, including ambulance, outpatient and inpatient services, and the operating room. The $5 \mathrm{G}$ smart hospital has several potential benefits, as outlined below.

In 2019, GD2H started to guide complex surgery conducted in remote hospitals, connected live via $5 \mathrm{G}$, that allowed the operating room to be turned into a classroom ${ }^{4}$. Since then, GD2H has continued to explore ways that $5 \mathrm{G}$ could overcome the problem of real-time data sharing due to distance and volume of data. GD2H has now equipped its ambulances with a portable computerized tomography scanner, an electrocardiogram and an echocardiogram machine, as well as first-aid supplies. Once a patient enters the ambulance, the use of $5 \mathrm{G}$ allows real-time data on rapid assessment, with examinations and diagnoses, monitoring, and initial treatments transmitted to the hospital system simultaneously. If necessary, a multidisciplinary team can arrive within minutes for consultation and decision-making, while the emergency room is made ready to receive the patient. The 5G-powered ambulance as a mini-hospital will shorten the time from 